\title{
Erratum to: Negative expertise: comparing differently tenured elder care nurses' negative knowledge
}

\author{
Martin Gartmeier • Erno Lehtinen • Hans Gruber • \\ Helmut Heid
}

Published online: 10 March 2011

(C) Instituto Superior de Psicologia Aplicada, Lisboa, Portugal and Springer Science+Business Media BV 2011

\section{Erratum to: Eur J Psychol Educ \\ DOI 10.1007/s10212-010-0042-5}

In the published version of the article "Negative Expertise: Comparing Differently Experienced Elder Care Nurses' Negative Knowledge" by Gartmeier, Lehtinen, Gruber and Heid, the legends to the Figs. 2, 3, 4 and 5 were incomplete. Following, the figures with complete captions are to be found.

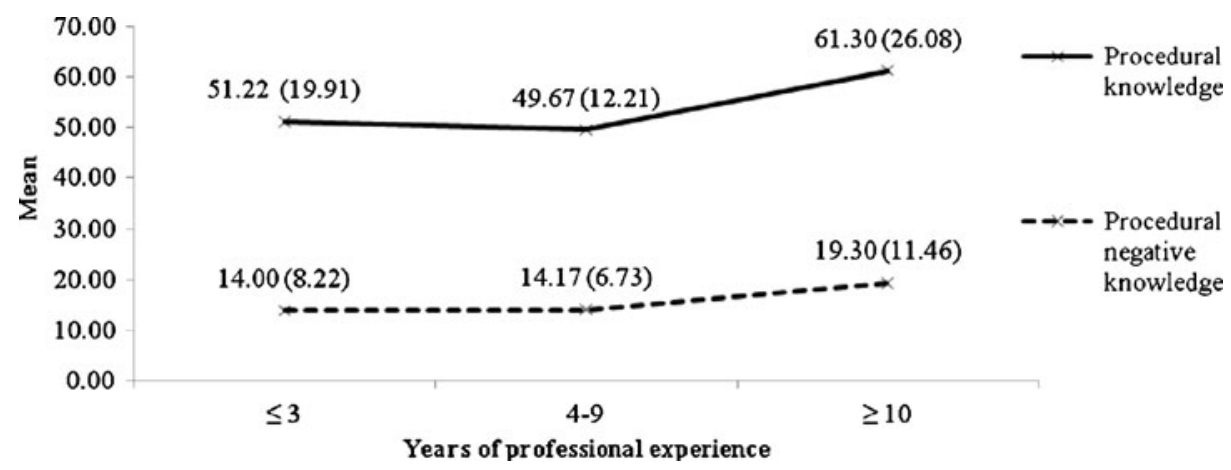

Fig. 2 Group differences in procedural knowledge and procedural negative knowledge. For each level of professional experience, the average amount of statements per participant and interview in the self-reflective and self-reflective negative focus is depicted (with standard deviations in brackets)

The online version of the original article can be found under http://dx.doi.org/10.1007/s10212-010-0042-5.

\footnotetext{
M. Gartmeier $(\bowtie)$

e-mail: martin.gartmeier@tum.de

E. Lehtinen

University of Turku, Turku, Finland

H. Gruber $\cdot$ H. Heid

University of Regensburg, Regensburg, Germany
}

TUM School of Education, Susanne Klatten Endowed Chair for Empirical Educational Research, Technische Universität München, Schellingstr. 33, 80799 München, Germany 


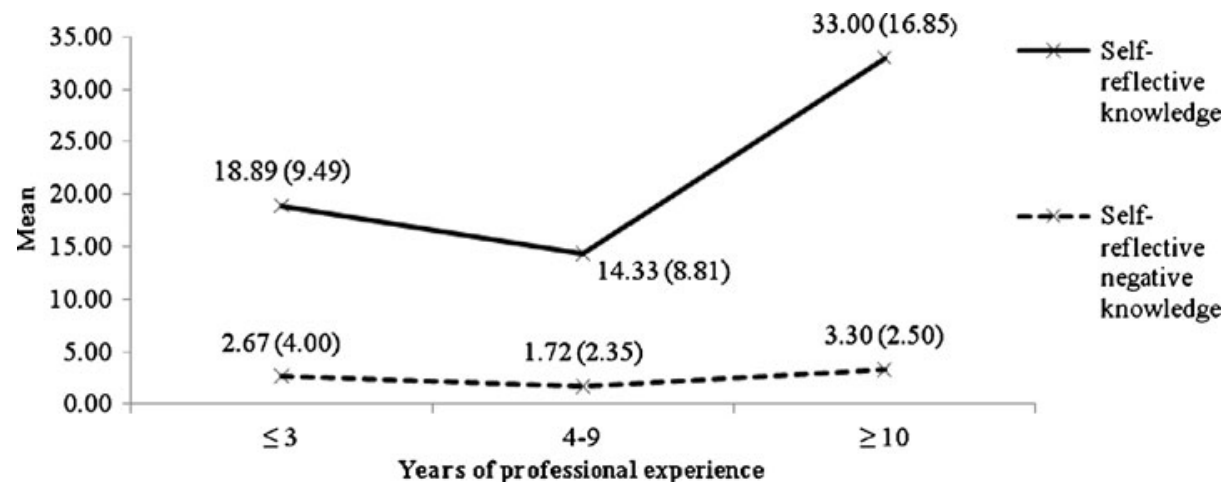

Fig. 3 Group differences in self-reflective knowledge and self-reflective negative knowledge. For each level of professional experience, the average amount of statements per participant and interview in the procedural and procedural negative focus is depicted (with standard deviations in brackets)

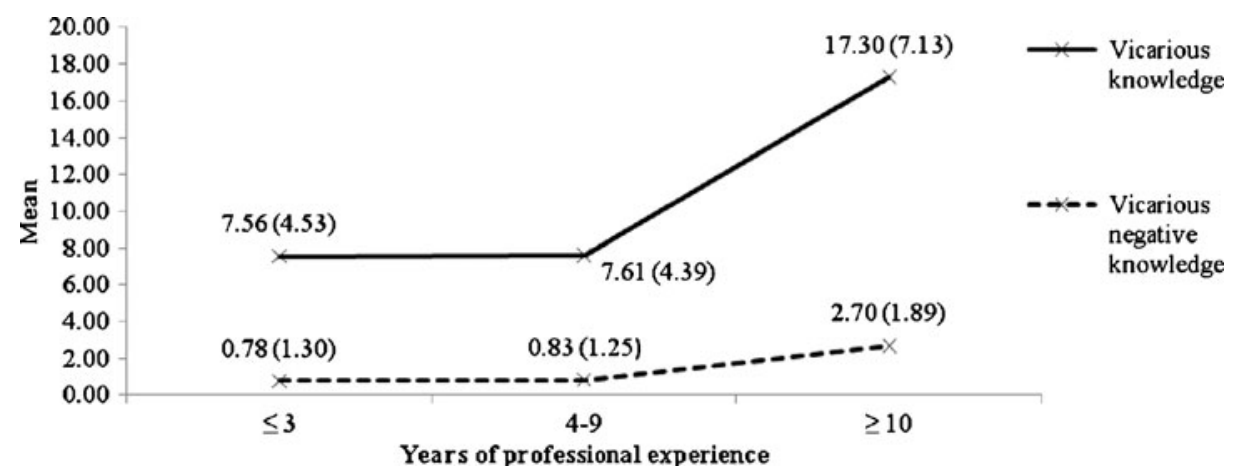

Fig. 4 Group differences in vicarious knowledge and vicarious negative knowledge. For each level of professional experience, the average amount of statements per participant and interview in the vicarious and vicarious negative focus is depicted (with standard deviations in brackets)

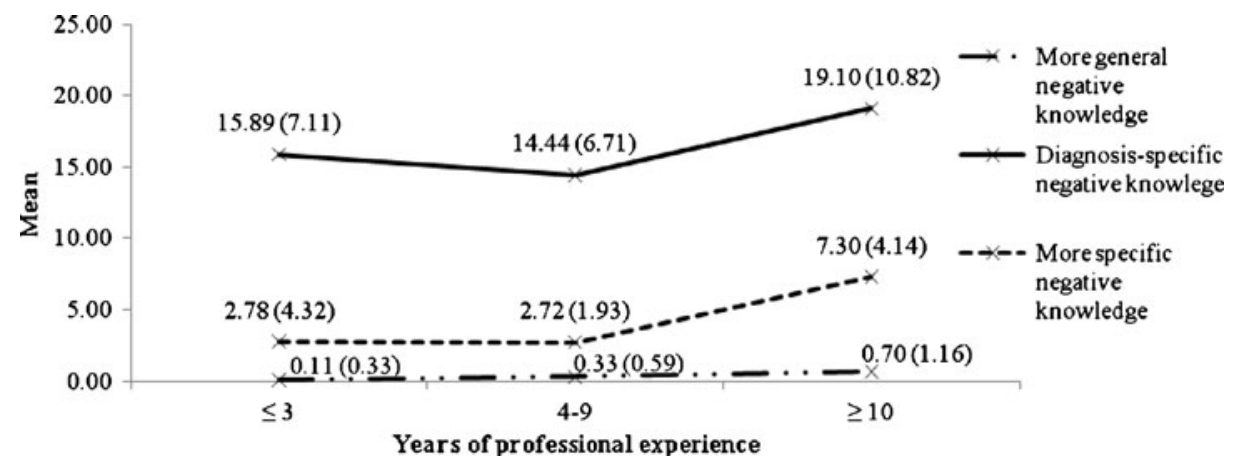

Fig. 5 Group differences in negative knowledge in different levels of specificity. For each level of professional experience, the average amount of statements of negative knowledge in the different levels of specificity is depicted per participant and interview (with standard deviations in brackets) 